\title{
Labyrinthe
}

$30 \mid 2008$ (2)

Ecologie $=X$

\section{De Frederick J. Turner à Donald Worster}

L'écologie dans la New Western History aux États-Unis

\section{François Duban}

\section{(2) OpenEdition \\ Journals}

Édition électronique

URL : https://journals.openedition.org/labyrinthe/3767

DOI : $10.4000 /$ labyrinthe.3767

ISSN : 1950-6031

Éditeur

Hermann

Édition imprimée

Date de publication : 10 juin 2008

Pagination : $37-47$

ISBN : 978-2-9526131-7-0

Référence électronique

François Duban, « De Frederick J. Turner à Donald Worster », Labyrinthe [En ligne], 30 | 2008 (2), mis en ligne le 27 mai 2008, consulté le 12 janvier 2022. URL : http://journals.openedition.org/labyrinthe/3767 ; DOI : https://doi.org/10.4000/labyrinthe.3767 


\title{
DE FREDERICK J. TURNER À DONALD WORSTER L'écologie dans la New Western History aux États-Unis
}

\author{
François DUBan \\ francois.duban@orange.fr
}

Aux États-Unis, dire l'histoire conduit à constater que nature et culture entretiennent dès la naissance de l'Amérique blanche un dialogue ambivalent: à la crainte puritaine de la wilderness, nature sauvage hostile à conquérir puis à dominer, répond l'attrait des pionniers pour les terres et les richesses de l'inconnu à l'Ouest, et plus tard du grand public pour des paysages grandioses à préserver. De nos jours, ce dialogue ininterrompu se nourrit du discours écologiste. L'écologie en tant que science, qui s'est développée notamment aux États-Unis au XIX ${ }^{e}$ siècle, et ses avatars, toujours aux États-Unis - environnementalisme, philosophies de la nature comme l'écologie profonde - ont eu un impact déterminant sur l'historiographie de l'Ouest, région centrale dans l'imaginaire américain depuis toujours. La place de l'Ouest dans les grands mythes nationaux fut renforcée et légitimée par l'historien Frederick Jackson Turner dont l'œuvre occultait toutefois les aspects moins glorieux de l'avancée de «la» civilisation au-delà des Appalaches ${ }^{1}$.

Turner a consacré ses travaux à l'étude de ce qu'il est convenu d'appeler la Frontière, au sens américain du terme, le front où la civilisation rencontrait la sauvagerie, zone mouvante entre nature sauvage où s'étendaient les terres «libres » à l'ouest, et les États déjà constitués à l'est, processus - et Turner insiste sur ce terme (process) - qui prend fin officiellement en 1890. Turner parle de l'Ouest comme du creuset de l'identité américaine, là où se sont forgés les grands traits de l'américanité et affirme que la démocratie américaine n'est pas l'héritière des penseurs européens ou des traditions anglo-saxonnes, mais qu'elle

1. L'article majeur de Frederick J. Turner est $\propto$ The Significance of History $n$ [1893]; reproduit dans History, Frontier, and Section, Albuquerque, University of New Mexico Press, 1993. 
résulte des conditions de vie sur la Frontière où chacun arrivait avec les mêmes chances sur une terre réputée vierge, aux ressources abondantes - conditions nécessaires, selon lui, au développement de la société démocratique américaine. L'Ouest devenait le jardin du monde. Pour Turner, le contact du pionnier avec la nature lui redonnait une innocence première ainsi que la vigueur des peuples sauvages, et l'impression de vivre en harmonie avec la nature, loin des maux de la Révolution industrielle.

Cette thèse de la Frontière (frontier thesis) a connu un retentissement extraordinaire. Cela peut notamment s'expliquer par la place accordée dans la thèse turnérienne aux mythes nationaux fondateurs hérités de l'agrarianisme jeffersonien. Véritable utopie aux yeux des Européens, l'instauration de la démocratie sur le continent américain passait, selon Jefferson, par la dissolution de la corruption de la classe politique qui, dans le passé, avait conduit les républiques à leur fin. Considérant la nature comme source de vertu, Jefferson mit ainsi en place une doctrine, l'agrarianisme, et un appareil législatif approprié, la Land Ordinance (1785), pour faire des citoyens américains de petits fermiers libres et vertueux. Les terres publiques acquises à l'ouest seraient ouvertes à la colonisation, et des générations de pionniers deviendraient ainsi les gardiens de la démocratie. Cet idéal agrarien devait perdurer dans la conscience collective jusqu'au début du $\mathrm{XX}^{\mathrm{e}}$ siècle, à l'époque même où Turner, dont la thèse de la Frontière conforte l'héritage jeffersonien ainsi que le nationalisme contemporain, connaît son plus grand succès.

À la suite des travaux d'Henri Nash Smith, qui dénonce dans Virgin Land $^{2}$ l'adéquation entre les mythes nationaux de l'Amérique et les conceptions turnériennes, Turner est l'objet de vives critiques qui prennent toute leur ampleur dans les années 1960-1970. Alors qu'émergent de nouvelles approches disciplinaires tels que les postcolonial ou les women studies, on reproche notamment à Turner d'avoir occulté les spoliations et les violences infligées aux minorités ethniques par la race blanche et de n'avoir rien ou trop peu dit du rôle des femmes dans l'histoire de l'Ouest, ou de celui des Indiens, des Mexicains, et des immigrants venus d'Asie. En outre, que Turner se soit donné comme modèle

2. Cambridge, Harvard University Press, 1950. 
explicatif un processus qui s'arrête en 1890 laisse a priori l'historien démuni pour dire l'histoire de l'Ouest aux siècles suivants.

La critique de l'œuvre de Turner a ainsi fédéré une génération d'historiens qui fondent la «Nouvelle Histoire de l'Ouest» (New Western History). L'une de ses principales représentantes, Patricia Nelson Limerick, a tenté de la définir à partir de la notion d'un Ouest en tant que région, située au-delà du centième méridien. La frontière ne s'arrête ni en 1890, ni en 1893, ni en aucune autre année. Il n'est plus question d'un seul processus à l'œuvre dans la mise en valeur des terres, mais de plusieurs, dont l'invasion, la conquête, la colonisation et l'exploitation. Le «progrès" de «la" civilisation - un thème qui domine la pensée de Turner - au-delà du Mississippi est remis en question, et la possibilité de l'existence du mal infligé à d'autres, de l'échec (tellement étranger à la culture américaine de la réussite), est prise en considération. De plus, ces «nouveaux historiens» renoncent à l'idée d'une « objectivité omnisciente et neutre ${ }^{3}$ » et développent leurs recherches en assumant un possible engagement moral, idéologique sinon politique.

Si Patricia Limerick a mené l'attaque frontale la plus reconnue contre l'héritage de Turner, son nom se trouve fréquemment associé à ceux de trois autres historiens qui, ensemble, ont propulsé la Nouvelle Histoire de l'Ouest jusque dans les médias et forment ce qu'on appelle aujourd'hui communément la «bande des quatre» (The Gang of Four): William Cronon, Richard White et surtout Donald Worster $r^{4}$, dont le cas nous intéresse ici en particulier, pour s'être pleinement associé aux critiques à l'égard de Turner dans une perspective environnementale, en dénonçant par ce biais la conception «fumeuse» (vaporous) d'un Ouest en tant que simple progression d'une zone de contact ${ }^{5}$.

3. Patricia Limerick, citée par Derek Larson, «What is the New Western History? n, http://employees.csbsju.edu/dlarson/Writings/HTML\%20copies/New\%20Western\%20History.htm\#N_21_ [consulté le 15 avril 2007].

4. Ces auteurs doivent leur notoriété aux ouvrages suivants: Donald Worster, Rivers of Empire, New York, Pantheon Books, 1985; William Cronon, Nature's Metropolis, New York, W.W. Norton, 1991; Richard White, "It's Your Misfortune and None of My Own ", Norman, University of Oklahoma Press, 1991. Le livre de Patricia Nelson Limerick, The Legacy of Conquest, New York, W.W. Norton, 1987, est souvent cité comme l'œuvre centrale de la Nouvelle Histoire de l'Ouest.

5. Voir Worster, Under Western Skies, New York, Oxford University Press, 1992, p. 23 [dorénavant noté UWS]. 
Ainsi, dans Rivers of Empire (1985) Worster met-il à mal la théorie turnérienne, «sans eau, sans aridité, sans emprise technologique, qui en vérité a très peu en elle de l'Ouest tel qu'on le définit aujourd'hui ${ }^{6}{ }$. Turner, né au Wisconsin, État suffisamment arrosé pour une agriculture de type européen, avait fait de la colonisation du Midwest son modèle, et n'avait pas visité l'Ouest, aride au-delà du centième méridien. Worster préfère rappeler que l'hubris dont fait montre l'espèce bipède dans l'Ouest se heurtera toujours à une réalité incontournable: «Tant que l'Ouest n'aura pas découvert comment produire [l'eau] en abondance et sans contrainte, il lui faudra continuer à se plier aux exigences de la nature" $[U W S$, p. 32]. Il trouve également à redire au silence turnérien sur la violence faite aux minorités et à la nature par l'industrie pétrolière et minière, les sabots des troupeaux des ranchers sur les terres publiques, l'irrigation à grande échelle. Worster relève en revanche que le mythe turnérien suggère un retour à la nature, l'occasion de retrouver la santé et le sens d'une harmonie avec le monde loin des discordances de l'univers des usines, de la technologie, des taudis urbains, de la pauvreté.

\section{Donald Worster et l'histoire environnementale}

Huit années avant Rivers of Empire, Worster a publié un ouvrage consacré à l'histoire des représentations de la nature en Occident, Nature's Economy: A History of Ecological Ideas ${ }^{7}$. Il est permis de s'interroger sur les raisons qui ont poussé Worster à entreprendre un tel travail. Son auteur explique que la seconde moitié du $\mathrm{XX}^{\mathrm{e}}$ siècle étant dominée par l'écologie, au point qu'il soit question de l'appeler «Âge de l'écologie», il voulait non pas étudier les raisons de cet état de fait, mais explorer l'évolution des idées en lien avec la nature depuis l'histoire naturelle du XVIII' siècle. Assurément, note Worster, la représentation que l'homme se donne de la nature a des conséquences sur sa relation au monde naturel. Ainsi, pour remédier aux dégradations infligées à ce monde, nous en remettons-nous de plus en plus à l'écologie en tant que science pour nous guider $[N E, \mathrm{p}$. x]. Mais Worster se défend de

6. Op. cit., p. 11.

7. Cambridge University Press, Cambridge, 1977 [dorénavant noté NE]. 
faire une histoire de la science, et affirme plutôt vouloir suivre l'histoire des idées; il n'y a pas en écologie, constate-t-il, de progrès des connaissances comme dans les sciences dures. Il s'intéresse moins à la science de l'écologique qu'à la pensée écologique et à ses ramifications littéraires, économiques, philosophiques (Worster parle de la "grande ombre» - larger penumbra - de la pensée écologique). Nous retiendrons de ces propos prudents que l'historien a mené une enquête approfondie sur les fondements d'une science dont il souhaite faire un outil. Mais de ce corps de savoir sans contours bien définis, on peut se demander s'il s'agit d'une science stricto sensu ou d'une représentation en perpétuelle évolution du monde naturel.

Worster n'est pas le premier historien à s'intéresser à l'écologie et ses concepts. Roderick F. Nash, dont l'ouvrage Wilderness and the American Mind a fait date ${ }^{8}$, écrivait, en 1972: "L'historien environnemental comme l'écologue devraient penser en termes de touts, de communautés, d'interrelations et d'équilibres ${ }^{9} »$. Son livre The Rights of Nature (1989), en posant la question des droits de la nature, contribue à développer le courant contemporain de l'éthique environnementale. Écologie et éthique se trouvent ainsi associées, ce qui peut-être considéré comme une caractéristique de l'histoire environnementale américaine. Nash fait partie de ces universitaires qui, dans les années 1960 à 1980, ont bouleversé le paysage académique outre-Atlantique et contribué à l'arrivée de la political correctness des années 1990, mais aussi au développement de nouvelles disciplines dont les gay-lesbian studies, les gender studies, les cultural studies et les environmental studies. La New Left, la déroute au Vietnam, le Watergate avaient ébranlé un édifice que les déconstructionnistes n'ont pas fini de mettre à mal.

C'est dans ce contexte que les apports de la pensée écologique ont influencé l'historiographie de l'Ouest américain. Une nouvelle sousdiscipline, I'histoire environnementale est devenue un terrain où se rencontrent les méthodes et approches de nombreuses autres disciplines. L'historien Richard White, pour étudier l'impact des hommes sur le milieu, fait appel aux géologues, aux anthropologues, aux ethnobota-

8. New Haven, Yale University Press, 1967.

9. «American Environmental History: a New Teaching Frontier ", Pacific Historical Review, vol. 41, $\mathrm{n}^{\circ} 3,1972$, p. 363. 
nistes, aux spécialistes des forêts, aux sociologues, etc. ${ }^{10}$ Worster, pour sa part, souligne que

[l'histoire environnementale] a résisté à la tentation d'entourer ses travaux de strictes barrières disciplinaires, ce qui la forcerait à concevoir ses propres méthodes d'analyse, ou à demander à toutes ces disciplines qui se recoupent de demeurer dans leurs champs propres. Chacune peut avoir sa tradition, certes, sa façon bien à elle d'aborder les problèmes. Mais nous vivons une époque marquée par l'interdépendance ${ }^{11}[\ldots]$.

Cette Nouvelle Histoire, qui se concentre sur le monde naturel et son évolution aux mains des hommes, parfois même sur l'impact des actions anthropiques sur une espèce ou un groupe d'espèces, prolongeait l'approche de la Nouvelle Histoire sociale qui faisait entendre la voix des gens ordinaires ${ }^{12}$. Toutes les minorités, parmi lesquelles aussi désormais les espèces animales et végétales, les rivières et les rochers avaient voix au chapitre. Le procès de l'anthropocentrisme par l'écologie profonde (philosophie écologique radicale apparue en 1973, qui prône une réduction sensible des impacts de l'espèce humaine sur la biosphère, et la reconnaissance des droits des espèces vivantes) avait fait école. Les Nouveaux Historiens prenaient le risque de «décentrer l'homme» pour «remettre en cause les Grands Partages qui clivent l'humanité des non-humains " $\rightarrow$ "Quelle éthique pour l'écologie ? « et $\rightarrow$ «Écologistes, encore un effort pour devenir antihumanistes...» $\nleftarrow$ :

Afin de $[\ldots]$ se montrer à la hauteur de leurs responsabilités en tant qu'intellectuels, les historiens de l'Ouest doivent étudier les autres peuples et apprendre d'eux; il leur faut essayer par exemple de regarder le passé tel que peut le voir un Indien américain. De façon plus radicale encore, ils doivent essayer d'étudier le comportement humain dans une perspective non humaine, le regarder pour ainsi dire comme le ferait le reste de la nature [UWS, p. 17].

10. Voir note 4, p. 39.

11. The End of the Earth, New York, Cambridge University Press, 1988, p. 305.

12. Thomas R. Cox, « Research Opportunities in Twentieth-Century Western History: Natural Resources and the Environment ", dans Gerald D. Nash and Richard W. Etulain, Researching Western History, Albuquerque, University of New Mexico Press, 1997, p. 29. 
Il ne faudrait pas pour autant faire de Worster un apôtre de l'écologie profonde ni un militant imbu d'une idéologie politiquement correcte. On note toutefois dans ses écrits un engagement éthique, où l'on pourrait voir la marque d'un certain humanisme, en dépit de ses positions en faveur du monde naturel et de sa critique tranchée à l'encontre de l'ordre socio-économique dominant, à savoir le capitalisme $\rightarrow$ «Écologistes, encore un effort pour devenir antihumanistes..." $\leftarrow$. Worster fait même du refus de cautionner les pouvoirs en place la caractéristique distinctive la plus importante de la Nouvelle Histoire de l'Ouest. L'historien doit s'en démarquer et se montrer critique de la société, de ses pratiques, de ses valeurs [UWS, p. 15-16].

Nous sommes loin de l'image reçue de l'historien académique, qui tente de dire l'histoire de façon aussi neutre et objective que possible. Worster va très loin dans ses propos, et dénonce ceux qui crient au scandale en pensant à leur carrière, à leur salaire, à leur poste, et craignent de se faire taxer d'idéologues [UWS, p. 15-16]. Il attend donc des historiens qu'ils se proclament les agents de la conscience morale de leur société [UWS, p. 17], qu'ils se débarrassent des mythes anciens, qu'ils découvrent une nouvelle identité régionale et un ensemble de valeurs (loyalties) «plus compatibles avec un sens planétaire de notre responsabilité écologique» [UWS, p. 17-18]. Dans sa critique de Luc Ferry et de sa préoccupation de sauver l'humanisme au sens traditionnel du terme, Worster semble aussi approuver les engagements des écologistes les plus extrémistes, témoignant par son parti pris idéologique - de la pensée écologique (scientifique) à la pensée écologiste (engagée) - d'une conception non neutre de l'écologie :

Ferry écrit que l'écologie est une force potentiellement révolutionnaire, et pourquoi pas? Il affirme que nous avons fait notre révolution il y a deux cents ans, la seule dont nous aurons jamais besoin. Les écologistes répliquent que les conditions environnementales allant se détériorant, elles ont rendu une nouvelle révolution nécessaire, et ils se demandent pourquoi notre époque ne devrait pas avoir la possibilité d'inventer de nouvelles éthiques et de nouvelles institutions. S'il est humain d'essayer d'échapper à la tradition, d'explorer de nouvelles frontières morales, alors les écologistes engagés sont, dans l'esprit sinon dans le dogme, les héritiers légitimes des Lumières ${ }^{13}$.

13. Donald Worster, «The Rights of Nature: Has Deep Ecology Gone Too Far? », Foreign Affairs, vol. $74, n^{\circ} 6$, novembre/décembre 1995 , p. 11. 


\section{L'attitude militante de Worster ou le dialogue entre écologie et histoire}

Comment analyser dès lors ce qui peut paraître comme un amalgame étrange entre science, histoire, et engagement moral qui caractérise Worster? Peut-on y voir l'influence d'une pensée écologique héritière de l'histoire naturelle du XVIII ${ }^{e}$ siècle et de son émerveillement devant les beautés et les mystères de la nature? Worster, et d'autres, ne se tournent-ils pas vers la morale et l'éthique pour protéger le monde naturel parce qu'ils n'ont pas trouvé dans la science la justification de leur action?

L'écologie en tant que science a effectivement dans ses premiers temps rendu compte d'un ordre du monde naturel, et les écologues américains, notamment Frederic Clements (1874-1945), ont largement contribué à conforter l'idée d'un ordre qui conduirait à l'équilibre. En étudiant la prairie américaine, Clements montre que celle-ci, lorsqu'elle est agressée, retrouve après une succession de stades intermédiaires, un équilibre final qui caractérise l'état dit «climacique» du système

$\rightarrow$ «Vers une notion écologique du paysage» $\leftarrow$. C'est là ce que Clements appelle un "superorganisme " - par ce concept, il entend tout système naturel parvenu au stade ultime de son développement. Ordre, équilibre, harmonie seraient donc les caractéristiques du vivant lorsqu'il n'est pas soumis aux agressions - une vision qui trouve son achèvement avec l'introduction en 1935 par Arthur Tansley du concept d' «écosystème».

Worster, dans le dernier chapitre de Nature's Economy, retrace les étapes qui ont conduit à élaborer les concepts fondateurs de l'écologie scientifique - symbiose, homéostasie, écosystème -, mais que la déconstruction postmoderne a mis à rude épreuve. C'est à William Drury et Ian Nisbet, écologues américains, que Worster attribue l'une des plus significatives remises en cause de la notion d'équilibre climacique. En 1973, ils montrent que les forêts de Nouvelle Angleterre sont en perpétuelle évolution, que le changement erratique et non l'équilibre caractériserait cette évolution. Même la notion de succession de stades vers un état d'équilibre et la pensée holistique qui la sous-tend sont remises en cause: les écosystèmes n'existent pas, il n'y a que la compétition entre individus et leurs rencontres stochastiques qui prévalent 
dans le monde. La symbiose, l'harmonie, l'équilibre font place au chaos, au hasard, à la compétition.

La théorie du chaos a pu séduire un monde désormais bien au-delà du désenchantement, qui, mis devant la réalité de la crise environnementale globale et subissant en même temps les méfaits de la globalisation, en est venu à accepter le risque comme mode de vie, et secrètement séduit par l'idée d'apocalypse, renonce à l'idée même de sauver ce qui resterait de nature ${ }^{14}$. Worster en son temps, a dû tirer les conclusions de ce constat d'une science écologique d'un type particulier qui n'avait pas l'assise des sciences dures, dont les concepts et les conclusions étaient remis en cause, et dont les idées qui en étaient issues, depuis l'aube de la modernité jusqu'au désespoir postmoderniste, semblaient épouser les interrogations des sociétés occidentales. On ne pouvait s'appuyer sur une écologie scientifique pour écrire l'histoire, comme l'avait souhaité Roderick F. Nash: une position imprégnée du goût amer de la «défaite de la science» que ne partage assurément pas Glen Love $\rightarrow$ «L'écocritique et la science: vers une consilience?» $\leftarrow$, qui s'inquiète de ce que la pensée postmoderne ait réduit la science à un discours comme un autre:

[cela] non parce que je me crois obligé de cautionner le rôle de la science comme moteur technologique de ce qui est perçu comme prospérité ou progrès, mais parce que je souhaite affirmer le rôle de la science - littéralement le savoir - comme nous révélant comment nous et la nature fonctionnons, afin que nous puissions mieux réfléchir à quel chemin suivre au milieu des défis environnementaux colossaux qui nous font face ${ }^{15}$.

Worster a pris toute la mesure de l'immense complexité du monde biologique qui semble défier la raison et la compréhension humaines [NE, p. 406]. Et l'histoire est à l'image de ce monde que nous décrit la science. L'histoire qui le passionne le plus est physique et écologique: c'est l'histoire de la terre, complexe comme seule la nature peut l'être, mais c'est aussi l'histoire des hommes interagissant avec la nature sur le continent nord-américain [UWS, p. vI]:

14. Frederick Buell, From Apocalypse to Way of Life, New York, Routledge, 2003, p. 225.

15. Glen Love, Practical Ecocriticism, Charlottesville, University of Virginia Press, 2003, p. 38. 
En d'autres termes, l'histoire de [l'Ouest] est d'abord et avant tout celle d'une écologie humaine en évolution. Une région prend forme à mesure que des gens essaient de tirer leur subsistance d'une portion de cette terre, à mesure qu'ils s'adaptent à ses ressources et à leurs limites. Ce que l'historien régionaliste doit s'efforcer de connaître en premier c'est comment un peuple ou des peuples se sont approprié une terre, et, ensuite, quelle représentation ils s'en sont donnée, et comment ils ont essayé d'en tirer profit. Il fera l'inventaire des techniques de survie qui ont été adoptées, des modes de travail, de l'organisation économique de leur société et des relations à l'intérieur de celle-ci [UWS, p. 27].

Il y a quelque ironie à constater que la nécessité d'une approche similaire, ce dialogue entre la terre et les hommes, avait en fait été pressentie par Turner; et Worster en convient, affirmant clairement que le fondateur de l'historiographie de l'Ouest avait été parmi les premiers à noter que l'aridité des régions où s'établissaient les pionniers à la fin du $\mathrm{XIX}^{\mathrm{e}}$ siècle mettrait un terme au mythe du pionnier isolé et conquérant. La mise en valeur de ces terres exigeait des travaux d'irrigation au-delà des moyens du simple fermier. Les conséquences environnementales et sociales de ces travaux d'irrigation sont d'ailleurs le socle de l'œuvre de Worster. Il rend ainsi hommage à la prescience turnérienne, à sa prise en compte du poids de l'environnement dans l'histoire des hommes ${ }^{16}$ et de la nécessité d'une approche pluridisciplinaire pour dire l'histoire de l'Ouest. Michael Allen opère pour sa part un renversement encore plus brutal, affirmant que Turner, dans son essai de 1891, «The Significance of History ", parlait en faveur d'une méthodologie historique globale, préfigurant les cultural studies américaines et même l'école française des Annales ${ }^{17}$.

\section{Conclusion}

En soi, l'engagement moral de Worster n'est pas une exception dans la longue tradition de la pensée écologiste américaine: l'éthique protestante panthéiste de John Muir, chef de file du mouvement préserva-

16. Donald Worster, Rivers of Empire, op. cit., p. 12.

17. Michael Allen, «The "New" Western History Stillborn », The Historian, vol. 57, $n^{\circ} 1,1994$, p. 201-208. 
tionniste à l'origine de la création des parcs nationaux, la Land Ethic d'Aldo Leopold dont Bérengère Hurand rappelle la prescription la plus célèbre $^{18}$, enfin, l'exigence de justice exprimée et par les tenants de l'écologie profonde à l'égard des droits des espèces vivantes à l'épanouissement (flourishing), et par les communautés de gens de couleur du mouvement appelé «Justice environnementale», en sont autant de témoins. Worster voit même dans cet engagement moral l'aboutissement du processus démocratique de la modernité:

[...] nous avons étendu notre protection à chaque espèce menacée, même la plus insignifiante ou la moins attirante... Tous ces efforts pour la préservation de la nature, pour protéger les grands comme les forts, les petits comme les faibles, les inconnus comme les plus emblématiques découlent des représentations du monde de la démocratie moderne. Nous n'avons pas seulement cherché à préserver la Nature sous toutes ses formes mais aussi à ouvrir ces lieux protégés à tous et à chacun parmi les humains, sans tenir compte de leur appartenance de classe ou leur couleur, beaucoup mieux que nos universités, nos country clubs ou nos résidences privées (gated communities). Dans ce mouvement démocratique de préservation, nous avons reconnu que notre obligation morale s'étend au-delà de l'espèce humaine. En bref, les Américains ont suivi les pas du jeune Muir sur la piste de l'exigence passionnée d'un égalitarisme élargi dans nos rapports à la terre' ${ }^{19}$.

Plus remarquable, en revanche, est le fait - et c'est ce que nous avons notamment souligné - que l'engagement de Worster marque un repli de ses ambitions en tant qu'historien: il s'est d'abord tourné vers la science, pour constater qu'elle ne fournit pas les certitudes nécessaires pour valider les mythes premiers de l'écologie et de l'écologisme; le voici alors qui prône une éthique environnementale. Peut-être cette recherche de vertus morales en écologie n'est-elle après tout, selon l'expression de Julien Delord, que «le symptôme d'une science peu assurée d'ellemême" $\rightarrow$ «La recherche écologique à l'épreuve de la philosophie de l'environnement» $\leftarrow$. Il manque sans doute à l'écologie un socle scientifique suffisamment solide pour écrire une histoire méthodologiquement construite. C'est dire que l'écologie est une «science» à part, holistique par nature, qui ne sait pas, entre ordre et chaos, dire l'ordre du monde.

18. Voir note 10, p. 97.

19. $\propto$ John Muir and the Modern: Passion for Nature », Environmental History, vol. 10, $n^{\circ} 1,2005$, p. 18. 